\title{
Modos de produção do cuidado e a universalidade do acesso - análise de orientações federais para o trabalho das equipes da APS no Brasil
}

\author{
Modes of care production and universal access - an analysis of \\ federal guidance on the work of Primary Healthcare teams in Brazil
}

Erica Lima Costa de Menezes (https://orcid.org/0000-0002-3966-6382) ${ }^{1}$

Marta Inez Machado Verdi (https://orcid.org/0000-0001-7090-9541) ${ }^{1}$

Magda Duarte dos Anjos Scherer (https://orcid.org/0000-0002-1465-7949) ${ }^{2}$

Mirelle Finkler (http://orcid.org/0000-0001-5764-9183) ${ }^{1}$

${ }^{1}$ Programa de Pós-

Graduação em Saúde Coletiva, Centro de Ciências da Saúde, Universidade Federal de Santa Catarina. R. Eng. Agronômico Andrei Cristian Ferreira s/n, Trindade. 88040-900 Florianópolis SC Brasil. ericalcmenezes@gmail.com ${ }^{2}$ Programa de PósGraduação em Saúde Coletiva, Faculdade de Ciências da Saúde, Universidade de Brasília. Brasília DF Brasil.

\begin{abstract}
This article analyzes federal guidance on the organization of primary healthcare work processes and the modes of production of care aimed at promoting universal access to services. A qualitative document analysis was undertaken of documents related to the National Health Policy, National Program for Improving Primary Care Access and Quality, More Doctors Program, and National Tele health Networks Brazil Program. Five thematic categories were defined for content analysis: staff training, valuing staff and work, organization of health actions for/with people and communities, complexity of healthcare and multiprofessional work, and collective construction of health as a right. The results show that advances were made in promoting universal access, particularly in the documents issued before 2015, with actions related to staff training, welcoming, the establishment of evaluation processes, and expansion of the scope of actions. However, there has been a shift towards the universal coverage model, as shown by measures encouraging reductions in the functional diversity of health teams and the flexibilization of working hours, hindering the implementation of the SUS as an inclusive social policy.

Key words Primary Healthcare, Access to health services, Healthcare, Universal coverage, Unified Health System
\end{abstract}

Resumo Objetivou-se analisar as orientações federais para organização do processo de trabalho na Atenção Primária à Saúde e os modos de produção do cuidado, no sentido de promover a universalidade de acesso aos serviços, por meio de análise documental em fontes primárias oficiais relacionadas à Política Nacional de Atenção Básica e aos Programas Nacional de Melhoria do Acesso e Qualidade, Mais Médicos e Telessaúde Brasil Redes. A análise originou cinco categorias: formação de trabalhadores na e para a APS; valorização do trabalhador e do trabalho; organização das ações de saúde para/com as pessoas e comunidades; complexidade do fazer em saúde e o trabalho multiprofissional e; a construção coletiva da saúde como direito. Os resultados apontam avanços na promoção da universalidade, especialmente nos documentos anteriores a 2015, por conta de ações relacionadas à formação profissional, ao acolhimento, ao estabelecimento de processos de avaliação e a ampliação do escopo de ações. Entretanto, há aproximações com a cobertura universal, evidenciadas pelo incentivo à implantação de equipes com redução da composição multiprofissional e flexibilização da carga horária, que dificultam a efetivação do SUS como política social inclusiva.

Palavras-chave Atenção Primária à Saúde, Acesso aos serviços de saúde, Assistência à saúde, Cobertura universal, Sistema Único de Saúde 


\section{Introdução}

Políticas públicas expressam não só prioridades governamentais, mas também as escolhas das sociedades sobre o tipo de nação que pretendem construir. Refletem a orientação do Estado, traduzida em normas, leis, diretrizes, procedimentos e estratégias, bem como os valores considerados prioritários para a vida no coletivo. Uma sociedade constitui e é constituída pela forma como fundamenta as bases conceituais e legais-normativas de suas políticas públicas ${ }^{1}$. O Sistema Único de Saúde (SUS), principal política pública de saúde do Estado brasileiro, instituído pela Constituição Federal de 1988 como política social que deve garantir acesso universal e igualitário às ações e serviços de saúde para sua promoção, proteção e recuperação, foi embasado nos valores da justiça social e da solidariedade, considerando-se os cidadãos brasileiros sujeitos de direitos ${ }^{2}$.

Da concepção e formulação de uma política à sua implantação e execução, diversos aspectos - políticos, sociais, econômicos, institucionais, ideológicos, técnicos e culturais - estão envolvidos $^{1}$. Nesse processo, participam atores que colocam em disputa distintas concepções do direito à saúde, resultando em diferentes formas de acesso e utilização dos serviços. A depender do resultado dessas disputas, $\mathrm{o}$ acesso à saúde pode ser conduzido como "medida de caridade, benefício adquirido mediante pagamento prévio, usufruto de um direito de cidadania" ${ }^{1}$ ou, ainda, direito inerente à condição humana ${ }^{3}$. Essas distintas concepções orientam a elaboração das estratégias em cada governo, podendo ser contraditórias ao texto constitucional e demais bases legais fundantes do SUS.

Vivenciamos um período no qual medidas restritivas de austeridade fiscal são adotadas mundialmente, causando retrocessos e demarcando importantes limites aos avanços de políticas sociais. No Brasil, o congelamento das despesas primárias, a redução do gasto mínimo com saúde e educação e a realização de reformas estruturais $^{4}$, evidenciam direcionamentos que consideram os sistemas públicos e universais economicamente inviáveis, baseados na igualdade restrita de oportunidades, meritocracia e agência do mercado ${ }^{5}$.

Tais opções, ancoradas em uma matriz neoliberal, podem colocar em xeque a consolidação do princípio da universalidade do acesso que pressupõe a igualdade de direitos entre as pessoas, independentemente de classe social, econômica, gênero, cor/etnia, religião e da equidade como caminho para a igualdade social. Considerando esse cenário, é fundamental distinguir os conceitos de universalidade do acesso e de cobertura universal, que, por vezes, têm sido utilizados como sinônimos, mas que guardam diferenças importantes entre si. Na cobertura universal o objetivo é que todas as pessoas possam comprar algum tipo de seguro e, nele, o papel do Estado é restrito à regulação do sistema e à garantia de um mínimo para aqueles que não possam pagar pelos planos, sendo priorizados os valores do mercado e do individualismo. Modelo que ganhou reforço inclusive na recém-publicada Declaração de Astana, elaborada na Conferência Mundial sobre Atenção Primária à Saúde ${ }^{6}$.

No processo de construção do SUS houve ampliação do acesso aos serviços de saúde, com repercussões positivas no perfil epidemiológico; redução do número de contratos de trabalho temporários; investimento no provimento e qualificação dos trabalhadores de saúde; promoção e formalização da participação social; e aumento da conscientização da população sobre o direito à saúde vinculado à cidadania ${ }^{7}$. Não obstante, $\mathrm{o}$ SUS idealizado não tem encontrado ambiente fértil para sua consolidação, sofrendo de subfinanciamento desde sua implementação e com inúmeras e permanentes ameaças ${ }^{2}$.

A ordenação do SUS por meio da Atenção Primária à Saúde (APS), com adoção e ampliação da Estratégia Saúde da Família (ESF), tem como um dos objetivos a garantia da universalidade do acesso, entendido como a capacidade multidimensional dos serviços e sistemas de saúde de dar uma resposta resolutiva às necessidades de saúde apresentadas pelos usuários, configurandose como um dos pré-requisitos essenciais e parte constituinte de/para construção da atenção à saúde com qualidade ${ }^{8}$. Entretanto, isto requer, além da ampliação da cobertura das ações e serviços, o enfrentamento de desafios relacionados aos modos como as equipes produzem ações de saúde e às questões de gestão do trabalho ${ }^{2}$. Esses desafios exigem, daqueles que defendem o SUS, lutas constantes e avaliação permanente das políticas e programas implantados para correção de trajetória, qualificação e garantia da sua sustentabilidade:

Ao apresentar como a política de saúde [...] se constrói cotidianamente, é possivel compreender como a política social, em meio a ações técnicas, estratégias politicas e organizacionais, disputas por recursos e ideias, cumpre seu papel maior de proteger as coletividades ${ }^{1}$.

Considerando o papel fundamental do governo federal na formulação e condução das 


\section{Método}

Trata-se de pesquisa qualitativa que se valeu de análise documental ${ }^{9} \mathrm{em}$ fontes primárias que expressam e regulam a atual política sanitária brasileira. O corpus documental foi composto por 29 documentos oficiais relacionados à Política Nacional de Atenção Básica (PNAB), ao Programa Nacional de Melhoria do Acesso e Qualidade da Atenção Básica (PMAQ-AB), ao Programa Mais Médicos (PMM) e ao Programa Nacional Telessaúde Brasil Redes. Foram incluídas leis, portarias, informes, manuais e publicações técnicas do Departamento de Atenção Básica (DAB) e da Secretaria de Gestão do Trabalho e da Educação na Saúde (SGTES) do Ministério da Saúde, vinculadas a financiamento da gestão federal e publicadas no período de janeiro de 2011 a dezembro de 2017, cujos conteúdos apresentassem orientações para as práticas de saúde das equipes de APS (Quadro 1).

A opção pelo recorte em função do financiamento se deu por ser importante indutor da implantação de políticas pelos municípios. Foram excluídos políticas e programas relacionados aos agravos, ciclos de vidas e programas específicos. Para os documentos publicados em anos distintos, mas com semelhança de texto, foram considerados os dados do mais atual.

Todos os documentos foram localizados no website do Ministério da Saúde ${ }^{10}$, organizados e sistematizados em planilha eletrônica. Para operacionalizar a coleta e a análise dos dados, adotaram-se categorias pré-analíticas, adaptadas a partir dos elementos do trabalho e dos valores que influenciam a capacidade dos serviços em assegurar acesso, segundo Menezes et al. ${ }^{11,12}$ (Quadro 2).

Da análise do conteúdo dos documentos conformaram-se cinco categorias: (1) formação de trabalhadores na e para a APS; (2) valorização do trabalhador e do trabalho; (3) organização das ações de saúde para/com as pessoas e as comunidades; (4) complexidade do fazer em saúde e o trabalho multiprofissional; e (5) a construção coletiva da saúde como direito. Os resultados de cada categoria foram analisados à luz dos

referenciais teóricos da Bioética Cotidiana ${ }^{13}$ e da Ergologia ${ }^{14}$, os quais contribuíram na compreensão das políticas de saúde para a APS como componentes do conjunto de normas antecedentes acionadas e renormalizadas pelos trabalhadores diante do imprevisível da atividade de trabalho, assim como para reflexão sobre os valores presentes nos modos de produção do cuidado, que vão influenciar na garantia do princípio da universalidade do acesso.

\section{Resultados}

Formação de trabalhadores na e para a APS - O estímulo e a viabilização da formação para os trabalhadores de saúde são responsabilidades compartilhadas por todas as esferas de governo ${ }^{\mathrm{P} 1 \mathrm{P3}}$, razão pela qual as políticas analisadas orientam ações de Educação Permanente em Saúde (EPS) ${ }_{\mathrm{P} 1, \mathrm{P} 3, \mathrm{P} 8, \mathrm{P} 13}$, de Apoio Institucional $(\mathrm{AI})^{\mathrm{P} 1, \mathrm{P} 3, \mathrm{P}, \mathrm{P}, \mathrm{P} 8 \mathrm{P1}, \mathrm{P} 11}$, de Apoio Matricial (AM) $)^{\mathrm{P}, \mathrm{P} 3, \mathrm{P} 6, \mathrm{P} 11, \mathrm{P} 12}$, de Cooperação Horizontal ${ }^{\mathrm{P} 1, \mathrm{P} 3, \mathrm{P}, \mathrm{P}, \mathrm{P}, \mathrm{P} 10, \mathrm{P} 11}$ e de Supervisão acadêmica em serviço ${ }^{\mathrm{P} 16, \mathrm{P1} 7 \text {,P20-P23 }}$. Nesse contexto, destaca-se uma importante mudança, verificada na PNAB: a remoção da possibilidade de dedicação de até oito horas semanais do trabalho na ESF em cursos de especialização em saúde da família, em residências multiprofissionais e/ou de medicina de família e de comunidade (MFC), bem como em atividades de EPS e $\mathrm{AM}^{\mathrm{P} 1, \mathrm{P} 3}$.

Entre os diferentes modos de desenvolver ações de EPS elencadas nas políticas estão as estratégias educativas do Telessaúde ${ }^{\mathrm{P} 6, \mathrm{P} 7, \mathrm{P1} 1 \mathrm{P} 12, \mathrm{P} 17, \mathrm{P}-}$ 23-P25,P27-P29, a Comunidade de Práticas do Ministério da Saúde, os espaços para troca de experiências $^{\mathrm{P} 23, \mathrm{P} 25, \mathrm{P} 27, \mathrm{P} 29}$, a participação em cursos de atualização, qualificação e pós-graduação, os grupos de discussão, os sítios virtuais interativos ou redes sociais colaborativas e os momentos reservados nas reuniões periódicas da equi$\mathrm{pe}^{\mathrm{P} 6 \mathrm{P}, \mathrm{P}, \mathrm{P} 11 \mathrm{PP} 12}$.

Os documentos apresentam também propostas de ampliação e interiorização de vagas de ensino superior, e mudanças curriculares para os cursos de graduação e pós-graduação com o objetivo de formar profissionais com perfil adequado à Atenção Básica $(\mathrm{AB})^{\mathrm{P} 1, \mathrm{P} 3, \mathrm{P} 15, \mathrm{P} 16, \mathrm{P} 23}$. Ressalta-se a reordenação das vagas de cursos de medicina, com nítida priorização das regiões de saúde com menor relação de vagas e médicos por habitante, e o estabelecimento de novos parâmetros para a formação médica, com reserva de $30 \%$ da carga horária do internato desenvolvida na APS e em serviços de urgência e emergência do SUS $S^{\mathrm{P} 15, \mathrm{P} 16, \mathrm{P} 23}$. 


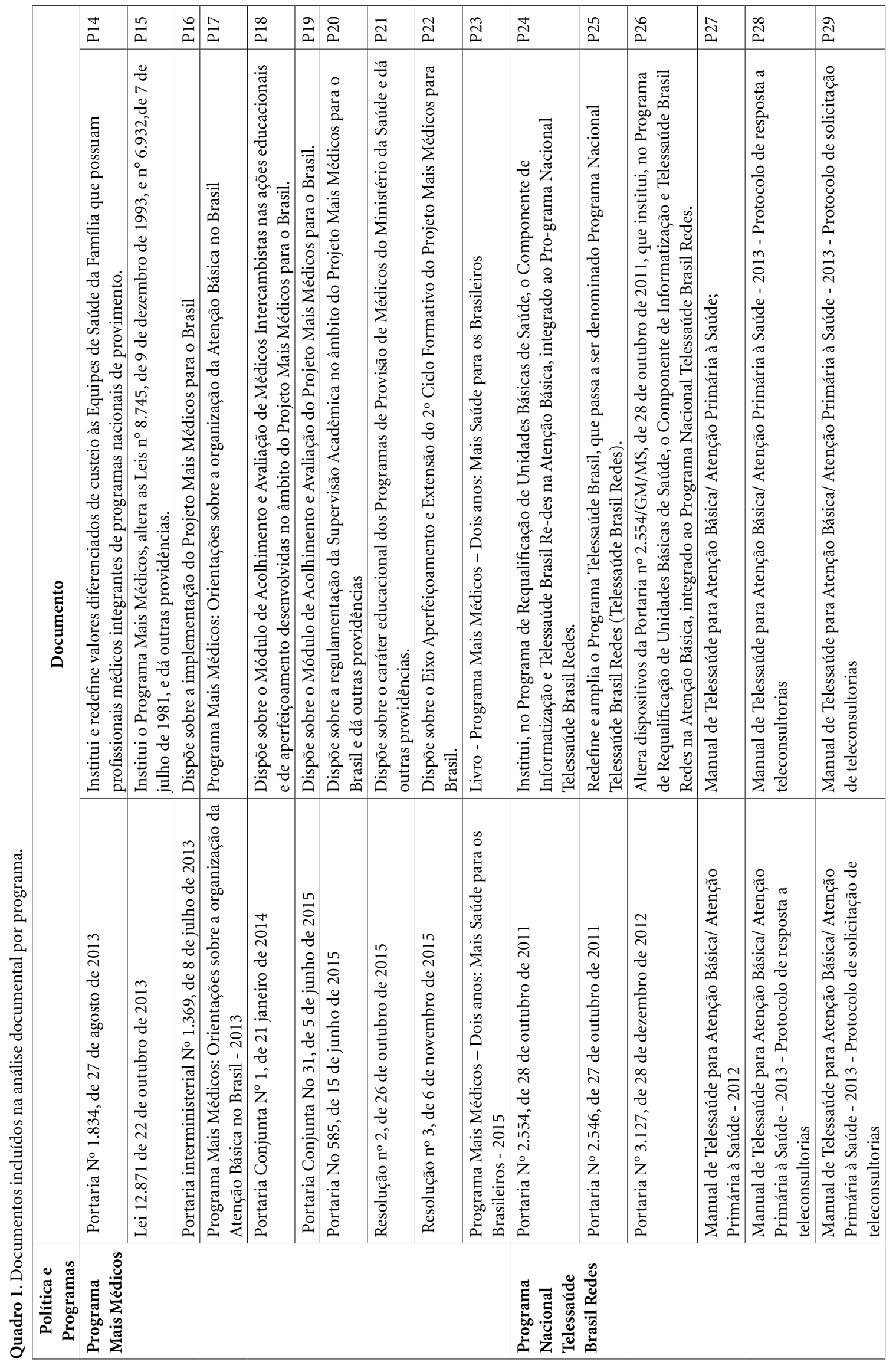


Quadro 2. Elementos que influenciam a capacidade dos serviços em assegurar acesso, agrupados em categorias pré-analíticas e fundamentados em valores.

\begin{tabular}{|c|c|c|}
\hline $\begin{array}{c}\text { Categorias } \\
\text { pré-analíticas }\end{array}$ & $\begin{array}{l}\text { Elementos que influenciam a capacidade dos serviços } \\
\text { em assegurar acesso }\end{array}$ & Valores \\
\hline \multirow{4}{*}{$\begin{array}{l}\text { Formação, } \\
\text { experiência e } \\
\text { domínio de } \\
\text { normas }\end{array}$} & Ofertas de Educação Permanente; & \multirow{4}{*}{ 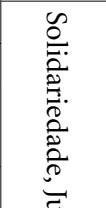 } \\
\hline & Formação na graduação; & \\
\hline & $\begin{array}{l}\text { Formações e normativas referentes ao trabalho na Atenção Primária à Saúde e } \\
\text { Estratégia Saúde da Família; }\end{array}$ & \\
\hline & Ofertas de apoio à prática cotidiana. & \\
\hline \multirow[t]{2}{*}{ Satisfação } & $\begin{array}{l}\text { Sobrecarga de trabalho: quantidade de trabalho diário; demanda para } \\
\text { atendimento; exigência de produtividade; }\end{array}$ & \multirow{7}{*}{ 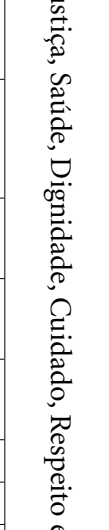 } \\
\hline & $\begin{array}{l}\text { Valorização: salários; vínculos de trabalho; mecanismos de valorização e } \\
\text { gratificação profissional; responsabilidade pelo cuidado das pessoas; imaginário } \\
\text { existente sobre os serviços públicos e importância do trabalho que realizam. }\end{array}$ & \\
\hline \multirow{5}{*}{$\begin{array}{l}\text { Gestão e } \\
\text { organização } \\
\text { do processo de } \\
\text { trabalho }\end{array}$} & $\begin{array}{l}\text { Territorialização: adscrição/adstrição das pessoas e famílias; cadastramento das } \\
\text { famílias; vínculação com local de residência/trabalho. }\end{array}$ & \\
\hline & $\begin{array}{l}\text { Agenda das equipes: organização da oferta; acolhimento com classificação de risco } \\
\text { x outros modos de marcação. }\end{array}$ & \\
\hline & $\begin{array}{l}\text { Ofertas de ações: visita domiciliar, atividades em grupo; atividades coletivas; apoio } \\
\text { matricial; }\end{array}$ & \\
\hline & Horário de funcionamento das UBS; & \\
\hline & Ambiência. & \\
\hline \multirow{3}{*}{$\begin{array}{l}\text { Trabalho } \\
\text { coletivo }\end{array}$} & Trabalho em equipe; & \multirow{7}{*}{ 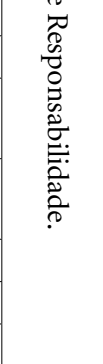 } \\
\hline & Oferta de ferramentas para o trabalho em equipe; & \\
\hline & $\begin{array}{l}\text { Composição das equipes: multiprofissionalidade, rotatividade, quantidade de } \\
\text { profissionais. }\end{array}$ & \\
\hline \multirow{4}{*}{$\begin{array}{l}\text { Integração / } \\
\text { interação com a } \\
\text { comunidade }\end{array}$} & $\begin{array}{l}\text { Respeito aos saberes, inclusão das singularidades; questões culturais e morais; } \\
\text { necessidade de saúde e conhecimento da realidade local; }\end{array}$ & \\
\hline & Escuta, vínculo, confiança, diálogo e a comunicação; & \\
\hline & Construção coletiva dos espaços e do planejamento em saúde; & \\
\hline & Participação popular/controle social. & \\
\hline
\end{tabular}

Adaptado de Menezes et al. ${ }^{11,12}$.

A inserção dos estudantes desde o início do curso nos serviços de saúde, a avaliação de progresso para alunos do $2^{\circ}, 4^{\circ}$ e $6^{\circ}$ anos, bem como as mudanças nos instrumentos de avaliação dos cursos e a criação do Contrato Organizativo de Ação Pública de Ensino-Saúde entre gestores loco-regionais do SUS e as instituições de ensino ${ }^{\mathrm{P} 23}$ foram outros aspectos relevantes encontrados. Cabe ainda destacar a ampliação de vagas de residência médica e multiprofissional, as reformulações curriculares e as relacionadas ao ingresso nas residências médicas ${ }^{\mathrm{P} 15, \mathrm{P} 23}$. $\mathrm{O}$ programa de residência em MFC passou a ter duração mínima de dois anos, sendo o primeiro obrigatório para o ingresso em alguns programas de residência médica. Já o PMM prevê ciclos formativos para profissionais médicos participantes, com módulo de acolhimento, especialização, aperfeiçoamento, extensão e supervisão ${ }^{\text {P19-P22 }}$.

Valorização do trabalhador e do trabalho - A garantia de direitos trabalhistas e previdenciários, a qualificação dos vínculos de trabalho, a implantação de carreiras que associem desenvolvimento do trabalhador com qualificação dos serviços, e a seleção, contratação e remuneração dos profissionais em conformidade com a legislação também são atribuições dos diferentes entes federados ${ }^{\mathrm{P} 1, \mathrm{P} 3}$. Em relação a estes elementos que influenciam o processo de trabalho das equipes da APS e, portanto, a universalidade do acesso, destaca-se a alteração do teto máximo de usuários a serem atendidos por equipe de Saúde da Família já em $2013^{\mathrm{P} 2}$ (e reafirmada na PNAB de $2017^{\mathrm{P} 3}$ ), permitindo assim a constituição de equi- 
pes responsáveis por até 2.000 pessoas, ainda que considerando que nas áreas mais vulneráveis a população deve ser menor.

Estabelecida pelo MS, a avaliação, a certificação e o pagamento por desempenho foi a estratégia estabelecida com intuito de ampliar e qualificar o acesso, por meio da adequação e a execução de padrões de boas práticas e organização das Unidades Básicas de Saúde (UBS) ${ }^{\mathrm{P} 6, \mathrm{P} 7, \mathrm{P} 11-13}$. Os padrões foram classificados nos processos avaliativos como obrigatórios; essenciais; estratégicos e gerais $^{\mathrm{P} 6, \mathrm{P} 7, \mathrm{P} 11-\mathrm{P} 13}$ e, na PNAB de 2017 , na diretriz organizativa, como essenciais e ampliados ${ }^{\mathrm{P} 3}$. Os instrumentos de avaliação elencam o conjunto de ações a serem desenvolvidas pelas equipes para garantir padrão de qualidade nacional ${ }^{\mathrm{P} 6, \mathrm{P} 7, \mathrm{P} 11-13}$. Neste contexto avaliativo, a remuneração e a premiação dos trabalhadores aparecem como instrumentos que consideram o desempenho de acordo com metas e resultados pactuados, além da progressão por antiguidade, por titulação e formação profissional e a possibilidade de distintas cargas horárias ${ }^{\mathrm{P} 6, \mathrm{P} 7, \mathrm{P} 11, \mathrm{P} 12}$.

Organização das ações de saúde para/com as pessoas e comunidades - A territorialização é trabalhada nas políticas analisadas tanto como diretriz organizacional, quanto como instrumento de planejamento das ações prioritárias em consonância com o princípio da equidade $\mathrm{e}^{\mathrm{P} 6 \mathrm{P} 11}$, por meio da definição do território, da classificação de risco e vulnerabilidade para a implantação das UBS, da redução das distâncias entre UBS e população, e do acompanhamento dinâmico da situação de saúde, considerando os aspectos geográficos, ambientais, sociais, econômicos, culturais, religiosos, demográficos e epidemiológi$\cos ^{\mathrm{P} 3, \mathrm{P} 6, \mathrm{P} 11}$.

Preconiza-se que a agenda seja formulada e compartilhada com base nas necessidades de saúde da população, contemplando as ações para grupos específicos e para demanda espontânea. O acolhimento, focado na demanda espontânea, é orientado a ser desenvolvido de forma humanizada, durante todo o período de funcionamento da UBS, com escuta qualificada, classificação de risco e vulnerabilidades, com utilização de protocolos e definição de critérios para agendamento de consultas ${ }^{\mathrm{P1}, \mathrm{P} 3, \mathrm{P} 6, \mathrm{P} 11}$. Pressupõe "uma lógica de organização e funcionamento do serviço de saúde que parte do princípio de que a UBS deva receber e ouvir todas as pessoas que a procuram, de modo universal e sem diferenciações excluden-

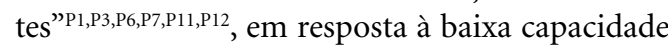
de atender os problemas agudos de saúde e com o objetivo de ampliar e qualificar o acesso ${ }^{\mathrm{P} 13}$.
Algumas das atribuições dos trabalhadores da APS, recorrentes nos documentos analisados, foram o atendimento clínico individual, pequenos procedimentos e coleta de exames, grupos terapêuticos, atividades comunitárias, práticas integrativas e complementares, atividades de educação e promoção da saúde, além de ações de vigilância, ações coletivas no território e reuniões de planejamento e avaliação ${ }^{\mathrm{P} 1, \mathrm{P} 3, \mathrm{P} 6, \mathrm{P} 7, \mathrm{P} 11, \mathrm{P} 12}$. Para os Agentes Comunitários de Saúde (ACS), foram incluídas, na PNAB de 2017, a aferição de pressão e temperatura, realização de medicação de glicemia e técnicas limpas de curativo, orientação e apoio em domicílio para a administração de medicação a usuários em situação de vulnerabilidade, desde que tenham formação e que seja respeitada autorização legal ${ }^{\mathrm{P} 3}$.

Embora recomende-se, em relação ao horário de funcionamento das UBS, o atendimento semanal de no mínimo 40 horas $^{\mathrm{P} 6, \mathrm{P} 11}$, cinco dias da semana e nos 12 meses do ano, foi flexibilizada a existência de horários alternativos de funcionamento, que atendam a necessidade da população que podem ser pactuados nas instâncias de participação social ${ }^{\mathrm{P} 3}$.

Ainda nesta categoria cabe mencionar as ações para a ambiência, relacionadas ao compromisso pretendido dos gestores em dotar as UBS com qualidade de equipamentos, infraestrutura e informatização com apoio do Governo Federal. Ações estas que podem ser identificadas no Programa de Requalificação ${ }^{\mathrm{P} 1, \mathrm{P} 3, \mathrm{P} 23, \mathrm{P} 24}$ e na compreensão das UBS como espaços de ensino-aprendizagem e de articulação entre ensino-serviço ${ }^{\mathrm{P} 23}$.

Complexidade do fazer em saúde e o trabalho multiprofissional - As políticas determinam o desenvolvimento da APS por meio do trabalho multiprofissional e interdisciplinar, entendendo que a ESF deve ser composta por médicos, enfermeiros, dentistas, auxiliar/técnico de saúde bucal, auxiliar/técnico de enfermagem e ACS, além de outras categorias profissionais que compõem o Núcleo Ampliado de Saúde da Família $(\mathrm{NASF})^{\mathrm{P} 1, \mathrm{P} 3}$. O A da sigla NASF teve seu sentido alterado de Apoio ${ }^{\mathrm{P1}}$ para Ampliado na última publicação da $\mathrm{PNAB}^{\mathrm{P} 3}$ e sua presença, como a da equipe de Saúde Bucal (eSB) é, desde então, facultada aos municípios ${ }^{\mathrm{P} 1, \mathrm{P} 3}$.

O financiamento de equipes transitórias para a ESF com flexibilização de carga horária ${ }^{\mathrm{P1} P 4, \mathrm{P} 5}$, ESF com apenas um ACS e equipe (eAB) composta apenas pela categoria médica e equipe de enfermagem ${ }^{\mathrm{P} 3}$, foram também possibilitados nesta revisão da PNAB. Outra flexibilização presente trata da orientação do número de ACS suficiente para cobrir $100 \%$ da população cadastrada ${ }^{\mathrm{P} 1}$ ape- 
nas para áreas de grande dispersão territorial, de risco e vulnerabilidade social ${ }^{\mathrm{P} 3}$.

O provimento de profissionais para a garantia de equipes completas, o investimento em formação para atuar em equipes multiprofissionais, o reconhecimento do residente como componente da equipe na $\operatorname{ESF}^{\mathrm{P} 23}$ e a inclusão de um gerente na $\mathrm{UBS}^{\mathrm{P} 3}$ são estratégias presentes nas políticas analisadas, capazes de contribuir para o fortalecimento do trabalho em equipe. Nesse mesmo sentido, algumas ferramentas e espaços são preconizados para o desenvolvimento do trabalho, como as consultas compartilhadas, a discussão de casos, a construção de planos terapêuticos, a definição de fluxos e atribuições de cada profissional, o monitoramento e a avaliação de resultados da atenção compartilhada ${ }^{\mathrm{P} 6 \mathrm{P} 11}$.

Construção coletiva da saúde como direito - O estímulo à participação popular e ao controle social aparece como atribuição e responsabilidade comum das diferentes esferas de governo, das equipes de saúde, por meio do incentivo à formação e participação nas reuniões dos conselhos locais e municipais de saúde. Um estímulo para a ampliação da autonomia e da capacidade de construção do cuidado em saúde $\mathrm{e}^{\mathrm{P1} 1 \mathrm{P} 3}$.

Ressalta-se a necessidade de conhecimento das demandas de saúde da população; do estabelecimento de relações de vínculo, de práticas dialógicas, críticas e reflexivas, da articulação do trabalho da equipe junto a cuidadores tradicionais; e do respeito às concepções morais e aos hábitos culturais das pessoas ${ }^{\mathrm{P} 6 \mathrm{P} 11}$, e se sugere que as equipes ofereçam formas e oportunidades para o registro da avaliação da satisfação dos usuários ${ }^{\mathrm{P} 3, \mathrm{P} 6 \mathrm{P} 11}$.

Finalmente, as políticas apontam como espaços para a consolidação da construção coletiva da saúde como um direito, os grupos de discussão de ações específicas, reuniões de conselhos e conferências locais/municipais de saúde e as reuniões periódicas de planejamento, acompanhamento e avaliação das ações de saúde, bem como a participação dos usuários do serviço ${ }^{\mathrm{P} 1, \mathrm{P} 3, \mathrm{P} 6, \mathrm{P7}}$.

\section{Discussão}

No processo analítico foi identificada a presença de ações relacionadas aos cinco componentes do trabalho que influenciam a capacidade dos serviços em assegurar acesso - formação, experiência e domínio das normas; satisfação; gestão e organização do processo de trabalho; trabalho coletivo; e integração/interação com a comunidade ${ }^{11,12}$ - demonstrando assim um reconhecimento dos desafios relativos à consolidação da universalidade do acesso nas políticas do setor. O Quadro 3 apresenta uma sistematização da análise desenvolvida, destacando os elementos que favorecem a universalidade do acesso e aqueles que o desfavorecem.

Os resultados apontam para o incentivo da EPS como conceito-estratégia para a qualificação dos trabalhadores e do cuidado, assim como o AI, o AM, a Cooperação Horizontal e a Supervisão Acadêmica. A formação desenvolvida no/ para o trabalho pode ajudar trabalhadores a refletirem sobre o que vivenciam no cotidiano das suas ações, possibilitando o desenvolvimento de competências necessárias à resolução dos problemas, discutindo as dificuldades e as alternativas e produzindo conhecimento ${ }^{15}$. Associadas às ações para trabalhar com os saberes produzidos nos atos de saúde, as políticas formulam estratégias para formação de trabalhadores, cujos saberes - científicos, acadêmicos, relativos à prática profissional, contribuam para a atuação na APS e na ESF, além de mudanças nas grades curriculares do ensino superior, com foco maior na formação médica.

De fato, é necessário desenvolver um caminho formativo calcado na inseparabilidade das ações dos profissionais de saúde dos interesses da coletividade, mudando a formação em saúde, tanto no plano da formação científica, quanto ético e política ${ }^{16}$. Por tal razão, "as transformações históricas das práticas em saúde e da formação médica ensejam a discussão da função das instituições de ensino para com a realidade sociossanitária e com o sistema de saúde no Brasil" ${ }^{17}$ e com a formação de profissionais socialmente responsáveis e competentes na construção da saúde como direito de cidadania. Para tanto, há que se ampliar o número de profissionais oriundos das diferentes classes sociais, pois "enquanto os médicos provirem somente de uma parte da população, a mais rica, será difícil, embora possível, cortar o cordão umbilical com a classe de origem e transformar a maneira tradicional de entender a medicina" ${ }^{16}$. A interiorização das universidades e as políticas afirmativas, como a de cota racial para ingresso no ensino superior, possivelmente tem contribuído para a mudança necessária do perfil dos profissionais de saúde.

Estes profissionais e a estruturação do sistema de saúde do qual participam, precisam ser capazes de enfrentar as questões cotidianas que afligem a maior parte da população, a despeito dos avanços tecnológicos da biomedicina que frequentemente tencionam as ações e os serviços de saúde para outros direcionamentos ${ }^{5}$. 
Quadro 3. Agrupamento dos elementos presentes nos documentos analisados em relação à universalidade do acesso, por categorias temáticas.

\begin{tabular}{|c|c|c|}
\hline $\begin{array}{l}\text { Categorias } \\
\text { temáticas }\end{array}$ & $\begin{array}{l}\text { Elementos que se aproximam da } \\
\text { universalidade do acesso }\end{array}$ & $\begin{array}{l}\text { Elementos que se afastam da universalidade } \\
\text { do acesso }\end{array}$ \\
\hline $\begin{array}{l}\text { A formação de } \\
\text { trabalhadores na e } \\
\text { para a APS }\end{array}$ & $\begin{array}{l}\text { Incentivo a formação desenvolvida no/ } \\
\text { para o trabalho; } \\
\text { Atividades desenvolvidas pelos } \\
\text { profissionais de saúde que consideram os } \\
\text { interesses da coletividade; } \\
\text { Mudança da formação médica; } \\
\text { Ampliação do número de profissionais } \\
\text { oriundos de diferentes classes sociais. }\end{array}$ & $\begin{array}{l}\text { A não garantia de horário reservado para } \\
\text { ações de educação permanente na Unidade de } \\
\text { Saúde; }\end{array}$ \\
\hline $\begin{array}{l}\text { A valorização do } \\
\text { trabalhador e do } \\
\text { trabalho }\end{array}$ & $\begin{array}{l}\text { A determinação de número máximo de } \\
\text { pessoas adscritas; } \\
\text { A redução no número de pessoas para o } \\
\text { cálculo do teto máximo de equipes. }\end{array}$ & $\begin{array}{l}\text { A flexibilização do quantitativo da população } \\
\text { adscrita para números superiores a } 3.500 ; \\
\text { Poucas ações relacionadas a desprecarização } \\
\text { dos vínculos trabalhistas. }\end{array}$ \\
\hline $\begin{array}{l}\text { Organizando } \\
\text { as ações de } \\
\text { saúde para/ } \\
\text { com as pessoas e } \\
\text { comunidades }\end{array}$ & $\begin{array}{l}\text { A ampliação do escopo de ações; } \\
\text { Categorização das ações em padrões } \\
\text { como critérios avaliativos e etapas a serem } \\
\text { alcançadas numa escala crescente de } \\
\text { qualificação dos serviços; } \\
\text { Organização da agenda considerando as } \\
\text { singularidades individuais e coletivas; } \\
\text { Acolhimento com ênfase no atendimento } \\
\text { à demanda espontânea. }\end{array}$ & $\begin{array}{l}\text { A ampliação do escopo de ações; } \\
\text { O número grande de ações, } \\
\text { O excesso de normas; } \\
\text { A exigência de produtividade; } \\
\text { Padrões como diretriz organizacional, } \\
\text { reduzindo a APS ao modelo seletivo e } \\
\text { fragmentado de cuidados mínimos à } \\
\text { população; Acolhimento restrito à demanda } \\
\text { espontânea. }\end{array}$ \\
\hline $\begin{array}{l}\text { As equipes } \\
\text { multiprofissionais } \\
\text { e a complexidade } \\
\text { do fazer em saúde }\end{array}$ & $\begin{array}{l}\text { A formação e ampliação de equipes } \\
\text { multiprofissionais; } \\
\text { Presença do gerente na unidade de saúde; } \\
\text { A flexibilização dos horários de } \\
\text { funcionamento das UBS e da carga } \\
\text { horária. }\end{array}$ & $\begin{array}{l}\text { Exigência de apenas um ACS para } \\
\text { financiamento, } \\
\text { A “integração" do trabalho do ACS e ACE, } \\
\text { podendo gerar sobrecarga; } \\
\text { O financiamento de eAB com apenas as } \\
\text { categorias médica e de enfermagem; } \\
\text { Alteração da denominação do NASF; } \\
\text { A inclusão de atribuições extras para os ACS e; } \\
\text { Flexibilização associada a redução de custos e } \\
\text { restrita as equipes de AB. }\end{array}$ \\
\hline $\begin{array}{l}\text { A construção } \\
\text { coletiva da saúde } \\
\text { como direito }\end{array}$ & $\begin{array}{l}\text { Incentivo ao controle social e participação } \\
\text { popular, a avaliação da satisfação das } \\
\text { pessoas com os profissionais e serviços e a } \\
\text { co-produção do cuidado. }\end{array}$ & $\begin{array}{l}\text { Ênfase nas estratégias de avaliação da } \\
\text { satisfação, por meio de pesquisas e consultas } \\
\text { e poucas orientações de como trabalhar a } \\
\text { participação popular. }\end{array}$ \\
\hline
\end{tabular}

Além da formação, a satisfação dos trabalhadores deve ser considerada para a garantia da qualificação e da ampliação do acesso. Estratégias para a melhoria e a regularização dos vínculos de trabalho foram encontradas nos resultados, mas ainda de forma incipiente. Houve redução na contratação do trabalho precário na ESF entre 2001 e 2009, com aumento da contratação protegida pelas prefeituras de médicos em $25 \%$, de dentistas em $40,8 \%$ e de ACS em $67,8 \%{ }^{18}$. Entretanto, a precarização dos vínculos de trabalho ainda se constitui como importante problema na maior parte dos municípios brasileiros. A terceirização da força de trabalho e a flexibilização dos vínculos trabalhistas na APS são mais presentes em municípios de maior porte populacional ${ }^{19}$. As portarias que tratam da PNAB, dos anos 2011 e 2017, passam longe do enfrentamento destes problemas, agravados pela flexibilização das jornadas de trabalho e da composição das equipes de saúde ${ }^{20}$.

A ampliação do escopo de ações e a organização da agenda deve considerar as singularidades individuais e coletivas. A categorização desse escopo em padrões, quando vistos como critérios avaliativos, pode servir como etapas a serem alcançadas numa escala crescente de qualificação dos serviços, contudo, enquanto diretriz organi- 
zacional, pode reduzir a APS ao modelo seletivo e fragmentado que oferta apenas os cuidados mínimos à população ${ }^{21}$.

Com relação à alteração da denominação do NASF e a inclusão de algumas atribuições aos ACS, há que se ter cautela, pois o discurso pela ampliação da resolubilidade da APS que acarreta priorização de práticas curativistas e ampliação da oferta de ações com menor custo e com equipes reduzidas, pode levar à sobrecarga e insatisfação dos trabalhadores e, consequentemente, a prejuízos no cuidado à saúde. A presença de gerentes nas UBS com formação adequada e com apoio da gestão municipal pode potencializar processos democráticos de produção da saúde.

A diversidade e a quantidade de ações a serem desenvolvidas, o excesso de normas, a exigência de produtividade e a padronização excessiva das ações levam à sobrecarga de trabalho ${ }^{22}$. Trabalhamos pela realização individual, em diversas dimensões, mas também para satisfazer as necessidades de grupos sociais ${ }^{23}$ em permanente dialética entre o que nos é exigido pelo meio e aquilo que consideramos ser o melhor ${ }^{24}$. Portanto, devese atentar à padronização excessiva, por seu risco de padronizar os seres no lugar das tecnologias ${ }^{25}$.

$\mathrm{O}$ acolhimento, com ênfase no atendimento à demanda espontânea contribui para a ampliação da clínica e contrapõe a ideia da APS com foco apenas nas ações de promoção da saúde e prevenção de agravos, o que resulta em redução do acesso e resolubilidade ${ }^{26}$. Entretanto, é preciso ampliar também os espaços para o acolhimento, não apenas daqueles que procuram as unidades de saúde, mas de toda a comunidade ${ }^{27}$.

A formação de equipes multiprofissionais reconhece essa complexidade, característica própria da organização da APS no Brasil. Reduzir a composição e a quantidade de equipes ameaça o conceito ampliado de saúde - pela tendência em focar nas questões médicas e biológicas - conceito este que pressupõe a sua concepção social, na qual tanto a saúde quanto a doença são culturalmente construídos e socialmente determinados ${ }^{5}$. Destacam-se três outros pontos articulados a essa análise: a possibilidade de formação de equipes de APS com a exigência de apenas um ACS para financiamento federal, a "integração" do trabalho do ACS e do Agente de Controle de Endemias (ACE) que pode gerar sobrecarga e/ou descaracterização da função dos mesmos, e o financiamento de equipes de saúde de $\mathrm{AB}$ com a presença apenas da categoria médica e da equipe de enfermagem.

As equipes com ACS em sua composição promovem a ampliação do vínculo, entre usuários e equipe, e facilitam o acesso ${ }^{28-30}$. A possibilidade de composição de equipes com apenas um ACS, a flexibilização da cobertura do cuidado por esses trabalhadores, a revogação da flexibilização da carga horária para os profissionais da ESF e a manutenção apenas para os profissionais das equipes de $\mathrm{AB}$, bem como a composição reduzida dessas, em tempos de políticas de austeridade, pode tornar a equipe de $\mathrm{AB}$ a primeira opção dos gestores, em detrimento a ESF ${ }^{21}$.

A flexibilização dos horários de funcionamento das UBS é uma necessidade sentida nos territórios, principalmente para atender as pessoas que trabalham no período comercial. Entretanto, se associada à flexibilização da carga horária dos profissionais e à redução de custos, a tendência é de reduzir o acesso, tanto em seus aspectos quantitativos como qualitativos, considerando a complexidade do fazer em saúde. Esse conjunto de flexibilizações em resposta à autonomia dos municípios para constituição de equipes de acordo com as singularidades e necessidades locais pode conflitar com uma atenção integral de qualidade.

O processo analítico evidenciou ainda a importância do incentivo ao controle social e à participação popular, a avaliação da satisfação das pessoas com os trabalhadores e serviços, e a coprodução do cuidado. Entretanto, as políticas pouco se referem aos modos de fazê-los. A construção do SUS como universal e público pressupõe a saúde como valor fundamental à vida e demanda um reconhecimento mútuo de se pertencer à mesma humanidade, ao contrário da convicção de ser possível usufruir do máximo de saúde, isolados do sofrimento alheio ${ }^{5}$. Assim, é importante que os trabalhadores contribuam efetivamente na construção de espaços e nos movimentos de controle social, ajudando a ressignificar o espaço público e político como um bem comum.

A equidade, consistentemente presente nos documentos analisados, está associada ao acesso universal como estratégia de redução das iniquidades em saúde, em direção à igualdade. Entretanto, é preciso atenção à "importância de medidas destinadas a remover os obstáculos ao bem-estar"3, pois oportunizar de igual modo não significa proporcionar as mesmas condições, nem tampouco ter como imagem-objetivo a radicalidade da igualdade de resultados.

Finalmente, cabe relacionar as políticas e os programas analisados ao conjunto de medidas adotadas pelos governos. Entre 2011 e 2015, os documentos descrevem ter havido aumento de 
mais de $100 \%$ no investimento para as APS, com objetivos declarados de consolidação da universalidade do acesso ao se preocupar não apenas com a ampliação deste, mas com a qualidade da atenção ${ }^{31}$. Já o governo interino que toma lugar após o impeachment presidencial em 2016, com posicionamento político-social marcadamente distinto, impõe medidas reducionistas ao SUS, ao mesmo tempo em que favorece o sistema suplementar, incentivando a criação de planos de saúde dito "populares" junto à iniciativa privada ${ }^{4}$.

Ao associar o direito à saúde à capacidade de pagamento pelos serviços, esse redirecionamento político, contrário ao SUS, reorienta o sistema do modelo que preconiza a universalidade do acesso ao de cobertura universal de saúde, "em que importa tão somente se a totalidade da população tem cobertura de algum serviço de saúde, independentemente se pago ou não e de sua capacidade de resolução do problema de saúde da pessoa" 4 .

Nesse contexto e com as novas regras fiscais estabelecidas pela Emenda Constitucional 95 de 2016, existirão maiores dificuldades para efetivação do direito à saúde no Brasil em decorrência da redução da despesa federal com o setor, mesmo com uma possível retomada do crescimento econômico, resultando em uma menor participação do Estado nas políticas sociais ${ }^{32}$.

\section{Considerações finais}

A universalidade do acesso à saúde pressupõe ações de ampliação da cobertura, como a estruturação das UBS, a garantia de insumos, a provisão e a adequada formação de trabalhadores, o apoio cotidiano para o enfrentamento das dificuldades e a identificação de alternativas, bem como a composição e o fazer das equipes multiprofissionais. Além disso, aspecto importante é a ampliação e a qualificação do acesso, tanto para grupos específicos, quanto para a demanda es- pontânea, incluindo a capacidade dos serviços de conhecer e atuar, considerando as necessidades de saúde das pessoas e comunidades, de forma cooperativa, participativa e inclusiva, efetivando a saúde como direito de cidadania.

A análise dos documentos sinalizou a presença de diversas proposições relacionadas ao enfrentamento das dificuldades para a garantia do acesso universal. Entretanto demonstram, ao mesmo tempo, o afastamento desta garantia ao reorientar o trabalho das equipes de saúde para o modelo de cobertura universal, principalmente nos documentos publicados após 2015. Entre os aspectos que atuam neste sentido, destaca-se a pouca ênfase às ações relacionadas à carreira profissional no SUS; o incentivo à implantação de equipes de $\mathrm{AB}$; a identificação de padrões essenciais e ampliados como diretriz organizacional do trabalho na APS; e a flexibilização do horário de funcionamento das UBS e do número máximo de pessoas sob a responsabilidade das equipes.

Evidenciou-se que havia tendência para a universalidade do acesso nos documentos anteriores a 2015 e para o modelo de cobertura universal posterior, ameaçando assim os avanços sociais conquistados nas primeiras três décadas do SUS. O governo que assume em 2016 implementa um conjunto de ações contrárias ao projeto eleito democraticamente, caminhando a passos largos em direção a um Estado mínimo ao reduzir investimentos na saúde e em outras políticas sociais, e ao ampliar o setor privado na área, que tem seus valores próprios que não os da solidariedade e justiça social.

Em uma sociedade verdadeiramente democrática, o direito à saúde deve ser universal, permanentemente defendido e priorizado. A construção de documentos institucionais que refletem políticas públicas voltadas à universalidade do acesso é fundamental, mas insuficiente para enfrentar os problemas do SUS que persistem desde sua origem e que se agravaram nos últimos anos. 


\section{Colaboradores}

ELC Menezes, MIM Verdi, MDA Scherer trabalharam em todas as etapas de elaboração do artigo; M Finkler trabalhou na revisão crítica e na aprovação da versão a ser publicada.

\section{Agradecimentos}

Ao Ministério da Ciência, Tecnologia e Inovação,Conselho Nacional de Desenvolvimento Científico e Tecnológico, Ministério da Saúde - Secretaria de Ciência, Tecnologia e Insumos Estratégicos - Departamento de Ciência e Tecnologia (MCTI/CNPq/MS - SCTIE - DECIT - Pesquisa em Saúde Bucal); à Coordenação de Aperfeiçoamento de Pessoal de Nível Superior - CAPES pela concessão da bolsa do Programa de Doutorado-sanduíche no Exterior (PDSE) à Erica Lima Costa de Menezes e à Fundação de Apoio à Pesquisa do Distrito Federal - FAP/DF pela concessão da bolsa de pós-doutorado sênior no exterior, à Magda Duarte dos Anjos Scherer.

\section{Referências}

1. Fleury S, Ouverney AM. Política de saúde: uma política social. In: Giovanella L, Escorel S, Lobato LVC, Noronha JC, Carvalho AI, organizadores. Politicas e sistema de saúde no Brasil. Rio de Janeiro: Editora Fiocruz; 2014. p. 25-57.

2. Paim JS. A Constituição Cidadã e os 25 anos do Sistema Único de Saúde (SUS). Cad Saude Publica [Internet]. 2013 Out [cited 2019 Jan 19]; 29(10):1927-1936. Available from: http://www.scielo.br/scielo.php?script=sci_arttext\&pid=S0102-311X2013001000003\&lng=en

3. Berlinguer G. Ética da Saúde. São Paulo: HUCITEC; 1996.

4. Vieira FS, Santos IS, Ocké-reis C, Rodrigues PHA. Politicas sociais e austeridade fiscal: como as politicas sociais são afetadas pelo austericídio da agenda neoliberal no Brasil e no mundo. Rio de Janeiro: CEBES; 2018.

5. Berlinguer G. Bioética Cotidiana. Brasília: Editora Universidade de Brasília; 2004.

6. World Health Organization (WHO). Declaração de Astana. [Internet]; 2018. [cited 2018 Dic 13]. Available from: https://www.who.int/docs/default-source/ primary-health/declaration/gcphc-declaration.pdf.

7. Paim JS, Travassos C, Almeida C, Bahia L, Macinko J. The Brazilian health system: history, advances, and challenges. Lancet 2011; 377(9779):1778-1797.

8. Assis MMA, JeSUS WLA. Acesso aos serviços de saúde: abordagens, conceitos, políticas e modelo de análise. Cien Saude Colet [Internet]. 2012 Nov [cited 2019 Nov 13]; 17(11):2865-2875. Available from: http://www.scielo.br/scielo.php?script=sci_arttext\&pid $=S 1413-81232012001100002 \& \operatorname{lng}=$ en

9. Sá-Silva JR, Almeida CD, Guindani JF. Pesquisa documental: pistas teóricas e metodológicas. Revista Brasileira de História \& Ciências Sociais [Internet]. 2009 Jul [cited 2018 Dic 10]; 1(1):1-15. Available from: https://www.rbhcs.com/rbhcs/article/view/6/pdf.

10. Brasil. Ministério da Saúde (MS). Portal do Ministério da Saúde. [Internet] 2018 [cited 2018 Jul 18]. Available from: http://portalms.saude.gov.br/.

11. Menezes ELC, Scherer MDA, Verdi MI, Pires DP. Modos de produzir cuidado e a universalidade do acesso na atenção primária à saúde. Saude Soc. [Internet]. 2017 Dez [cited 2019 Jan 19]; 26(4):888-903. Available from: http://www.scielo.br/scielo.php?script=sci_arttext\&pid=S0104-12902017000400888\&lng=pt

12. Menezes ELC, Scherer MDA, Ramos FRS. Influence of multiprofessional work on the production of care and access to primary health care. Rev. CEFAC [Internet]. 2018 May [cited 2019 Jan 19]; 20(3):342-352. Available from: http://www.scielo.br/scielo.php?script=sci_arttext\&pid=S1516-18462018000300342\&lng=en

13. Berlinguer G. Bioética Cotidiana. Brasília: Editora Universidade de Brasília; 2004.

14. Schwartz Y, Duc M, Durrive L. O Trabalho e Ergologia. In: Schwartz Y, Durrive L, organizadores. Trabalho e ergologia: conversas sobre atividade humana. Niterói: Ed. UFF; 2007. p. 24-46. 
15. Jacques A, Durrive L. O formador ergológico ou "Ergoformador": uma introdução a ergoformação. In: Schwartz Y, Durrive L, organizadores. Trabalho e ergologia: conversas sobre atividade humana. Niterói: Ed. UFF; 2007. p. 297-309.

16. Berlinguer G. Medicina e Política. São Paulo: CEBESHUCITEC; 1978.

17. Azevedo BMS, Ferigato S, Souza TP, Carvalho SR. A formação médica em debate: perspectivas a partir do encontro entre instituição de ensino e rede pública de saúde. Interface (Botucatu) [Internet]. $2013 \mathrm{Mar}$ [cited 2019 Jan 19]; 17(44):187-200. Available from: http://www.scielo.br/scielo.php?script=sci_arttext\&pid=S1414-32832013000100015\&lng=en. Epub Dec 11, 2012

18. Girardi S, Carvalho CL, Maas LWD, Farah J, Freire JA. O Trabalho Precário em Saúde: Tendências e Perspectivas na Estratégia da Saúde da Família. Saúde Debate [Internet]. 2010 maio [cited 2018 Aug 21]; (45):1123. Available from: http://epsm.nescon.medicina. ufmg.br/dialogos2/Biblioteca/Artigos_pdf/F17_O_ TRABALHO_PRECARIO_EM_SAUDE_TENDENCIAS\%20E\%20PERSPECTIVAS.pdf

19. Seidl H, Vieira SP, Fausto MCR, Lima RCD, Gagno J. Gestão do trabalho na Atenção Básica em Saúde: uma análise a partir da perspectiva das equipes participantes do PMAQ-AB. Saúde Debate [Internet]. 2014 Out [cited 2019 Jan 19]; 38(n. esp):94-108. Available from: http://www.scielo.br/scielo.php?script=sci_arttext\&pid=S0103-11042014000600094\&lng $=$ en

20. Narvai PC. Por uma carreira interfederativa, única e nacional do SUS. Abrasco [Internet]. 2017 [cited 2018 Sep 07]. Available from: https://www.abrasco.org.br/ site/outras-noticias/opiniao/por-uma-carreira-interfederativa-unica-e-nacional-do-SUS/31184/.

21. Morosini MVGC, Fonseca AF, Lima LD. Política Nacional de Atenção Básica 2017: retrocessos e riscos para o Sistema Único de Saúde. Saúde Debate [Internet]. 2018 Jan [cited 2019 Jan 19]; 42(116):11-24. Available from: http://www.scielo.br/scielo.php?script=sci_arttext\&pid=S0103-11042018000100011\&lng=en

22. Pires DEP, Machado RR, Soratto J, Scherer MA, Gonçalves ARS, Trindade LL. Nursing workloads in family health: implications for universal access. Rev. Latino-Am. Enfermagem [serial in Internet]. 2016 [cited 2017 May 4]; 24:e2682 Available in: https://www. revistas.usp.br/rlae/article/view/113366/111328

23. Schwart Y, Di Ruzza R, Le Bris R. Trabalho, emprego e cidadania. In: Schwartz Y, Durrive L. Trabalho e ergologia: conversas sobre atividade humana. Niterói: Ed. UFF; 2007. p. 278-295.

24. Schwartz Y. A comunidade científica ampliada e o regime de produção de saberes. Trabalho \& Educação [Internet]. $2000 \mathrm{Jul} / \mathrm{Dez}$ [cited $2018 \mathrm{Jul} 21$ ]; (7):38-46. Available from: https://seer.ufmg.br/index.php/trabe$\mathrm{du} /$ article/view/7592

25. Duraffourg J. Um robô, o trabalho e os queijos: algumas reflexões sobre o ponto de vista do trabalho. In: DIEESE, organizador. Emprego e desenvolvimento tecnológico: Brasil e contexto internacional. São Paulo: DIEESE; 1998. p. 123-144.
26. Tesser CD, Norman AH. Repensando o acesso ao cuidado na Estratégia Saúde da Família. Saúde Soc. [Internet]. 2014 Set [cited 2019 Sep 28]; 23(3):869883. Available from: http://www.scielo.br/scielo.php ?script $=$ sci_arttext $\&$ pid $=$ S0 10412902014000300 8698lng=en

27. Mitre SM, Andrade EIG, Cotta RMM. Avanços e desafios do acolhimento na operacionalização e qualificação do Sistema Único de Saúde na Atenção Primária: um resgate da produção bibliográfica do Brasil. Cien Saude Colet [Internet]. 2012 Ago [cited 2019 Jan 19]; 17(8):2071-2085. Available from: http://www.scielo.br/scielo.php?script=sci_arttex$\mathrm{t} \& \mathrm{pid}=$ S1413-81232012000800018\&lng $=$ en

28. Coimbra VCC, Oliveira MM, Kantorski LP, Heck RM, Jardim VMR, Ceolim T. Avaliação da estrutura - processo de acesso de usuários a uma Unidade de Saúde da Família. R. Pesq.: Cuid. Fundam. [Internet]. 2010 [cited 2017 Mar 01]; 2(3):1095-107. Available from: http://www.seer.unirio.br/index.php/cuidadofundamental/article/view/591/pdf_47

29. Lima SAV, Silva MRF, Carvalho EMF, Pessoa EAC, Brito ESV, Braga JPR. Elementos que influenciam o acesso à atenção primária na perspectiva dos profissionais e dos usuários de uma rede de serviços de saúde do Recife. Physis [Internet]. 2015 Jun [cited 2019 Jan 19]; 25(2):635-656. Available from: http://www.scielo .br/scielo.php?script=sci_arttext\&pid=S0103-7331 2015 000200635\&lng=en

30. Oliveira LS, Almeida LGN, Oliveira MAS, Gil GB, Alcione BOC, Medina MG, Pereira RAG. Acessibilidade a atenção básica em um distrito sanitário de Salvador. Cien Saude Colet [Internet]. 2012 Nov [cited 2019 Jan 19]; 17(11):3047-3056. Available from: http://www.scielo.br/scielo.php?script=sci_arttex$\mathrm{t} \&$ pid $=$ S1413-81232012001100021\&lng=en

31. Brasil. Ministério da Saúde (MS). Programa mais médicos - dois anos: mais saúde para os brasileiros. Brasília: MS; 2015.

32. Vieira FS, Benevides RPS. O Direito à Saúde no Brasil em Tempos de Crise Econômica, Ajuste Fiscal e Reforma Implícita do Estado. Revista de Estudos e Pesquisas sobre as Américas [Internet]. 2016 [cited 2018 Aug 01]; 10(3):1-28. Available from: http://webcache.googleusercontent.com/search?q=cache:R2-gjlDNm1YJ: periodicos.unb.br/index.php/repam/article/download/ $14896 / 13217 /+\& \mathrm{~cd}=5 \& \mathrm{hl}=\mathrm{pt}-\mathrm{BR} \& \mathrm{ct}=\mathrm{clnk} \& \mathrm{gl}=\mathrm{br} \&-$ client=firefox-b-ab.

Artigo apresentado em 30/05/2019

Aprovado em 07/08/2019

Versão final apresentada em 18/11/2019 
